X4. RÖNNE, HENNING, Copenhagen.- "Congenital Word-blindness in School Children," Trans. Ophthal. Soc. U.K. Vol. LVI, p. 311, 1936.

X5. ORton, SAmuel T., Iowa City, late of Columbia University.- "Wordblindness in School Children," Archives on. Neurology and Psychiatry, Vol. XIV, No. 5, November, 1925.

X5a. Marshall, Wallace, and Ferguson, John H.- "Hereditary Word. blindness as a Defect of Selective Association," The Jl. of Nervous and Mental Diseases, Vol. LXXXIX, No. 2, February, 1939.

X6. Ross Foundation.- The W. H. Ross Foundation for the Study of the Prevention of Blindness (Scotland).

X7. Macméken, M., Edinburgh University Educational Psychologist.- " Ocular Dominance in relation to Developmental Aphasia." Published by the Ross Foundation, May, 1939 "Developmental Aphasia in Educationally Retarded Children." Published by the Ross Foundation, May, 1942.

X8. BRUCE, AlexANDER, and DAwSON, JAMEs. " Multiple Neuromata of the Central Nervous System : their Structure and Histogenesis," Trans. Roy. Soc. Edinburgh, Vol. XLVIII, Pt. III, No. 27, 1913.

X9. MaRgulies.-Virchow's Archiv., Vol. CXCI, p. 94, 1908.

X10. BALLANCE and STEWART.-" "The Healing of Nerves," London, 1901.

X11. BERRY, RICHARD J. A.- " Some Recent Advances in the Study of Brain as the Implement of Mind," Trans. Roy. Soc. Edinburgh, 1944.

\title{
HISTOLOGICAL FINDINGS IN A CASE OF ANGIOID STREAKS
}

BY

\author{
F. H. VERHOEFF \\ BOSTON, MASS.
}

For the privilege of making this report I am indebted to the late Dr. Grady E. Clay. It deals with the microscopic examination of one eye removed after death from a patient with angioid streaks in both eyes. The eye was removed under the direction of Dr. Clay and sent by him to Dr. Algernon Reese for sectioning. A complete set of celloidin sections was prepared and stained under Dr. Reese's supervision and then sent to me at the request of Dr. Clay. The hospital at which the patient died was the Grady Memorial Hospital of Atlanta, Georgia, and to the authorities of this institution I am indebted for complete copies of the hospital records including the autopsy report on this patient. The clinical record was signed by Guh H. Adams, M.D. The autopsy was performed by Abner Golden, M.D., and the report signed by Walter H. Sheldon, M.D., pathologist. For the purposes of this paper the following summary of these hospital records will suffice :

The patient, a 50-year-old coloured female was admitted to the Grady Memorial Hospital, Atlanta, Ga., Jan. 28, 1944. She was known to have had syphilis which was inadequately treated. However, the Kahn test was negative at the final admission to the hospital although in 1935 the Wassermann test was 4 plus. She was known to have had hypertension since 1935. In 1939 the blood pressure was recorded as $200 / 110$ and at this time she began to experience intermittent oedema of the ankles. There were not marked symptoms, however, until three 
weeks before present admission when she became short of breath, orthopneic, and had several attacks of paroxysmal nocturnal dyspnea and a cough productive of thin white sputum. Peripheral edema became marked. The temperature was $100^{\circ}$, pulse 110 , respiration 30 , blood pressure $140 / 100$. The patient was in respiratory distress and the neck veins were distended. There was dullness to percussion over the right lung base where many medium and fine rales were heard. The heart was markedly enlarged to the left, and there was a systolic murmur at the apex. There was a diastolic gallop. The liver was enlarged to palpation, and tender. Reflexes were normal.

Dr. Clay observed and later reported to me a fact not noted in the hospital record, namely, that the patient had pseudo-xanthoma elasticum, a condition with which he was thoroughly familiar. On ophthalmoscopic examination Dr. Clay found a few angioid streaks in both fundi. He intended to give a detailed description of the streaks, but the patient died before he could do so. He recorded in addition that " the media were clear, the nerve heads normal. The retinal arterioles showed Grade 2, arteriosclerosis, and Grade 1 angiospasms."

The patient received digitalis and other cardiac therapy, but failed to respond. She died on her seventh hospital day.

At autopsy, extensive old and recent infarcts of the lungs were found. The branches of the pulmonary artery showed old and recent thrombosis, and some showed complete recanalization. The heart showed extensive old and recent infarction, and mural thrombi, displaying varying degrees of organization were present in both ventricles. The heart weighed $580 \mathrm{gms}$. and was dilated. The aorta throughout its length displayed a rather marked degree of arteriosclerosis and numerous calcified atheromatous plaques. The viscera showed chronic passive congestion and there was extensive central haemorrhage and necrosis of the liver, and recent infarction of the spleen. Of interest also, was thrombosis of the right transverse sinus of the dura mater.

Incidental findings included leiomyomata of the uterus and a small paraovarian cyst. Microscopic examination of the heart, lungs, spleen, pancreas, liver, kidneys, showed generalised arteriosclerosis. The sclerosis included branches of the coronary arteries.

Cultures obtained at autopsy showed no growth from the lungs and coagulase negative staphylococcus aureus from the blood.

The left eye, removed 11 hours after death, was fixed in 10 per cent. formalin. The celloidin sections made horizontally through the entire eye included the whole of the optic disc and a considerable extent of the fundus above and below it. The sections were thin, well stained in alum haematoxylin and eosin, and unusually free from artefacts. After careful examination, selected sections were dismounted to me and restained by my elastic tissue stain ${ }^{1}$ and by other methods.

The globe shows no distortion in the sections, and the choroid and retina are everywhere in situ except for a slight artificial separation of the retina anterior to the equator on the temporal side. The optic nerve and disc are normal. At the margin of the disc there are two large conglomerate colloid excrescences, each about $0.10 \mathrm{~mm}$. in diameter, one on the nasal side in the mid-plane, the other on the temporal side below. The former contains two minute blood vessels (Fig. 1). Otherwise the retina is normal. There is marked peripheral cystoid degeneration, but this is no greater than that often seen at this age. The rods and cones show the usual distortion seen in normal eyes removed post mortem. The choroid is so slightly shrunken by the fixation that the details 
of its structure are easily seen. It is normal except for the changes in Bruch's membrane next described, and a few insignificant foci - of lymphocytes. The elastic tissue of its stroma is neither increased nor diminished in amount, and stains normally. The

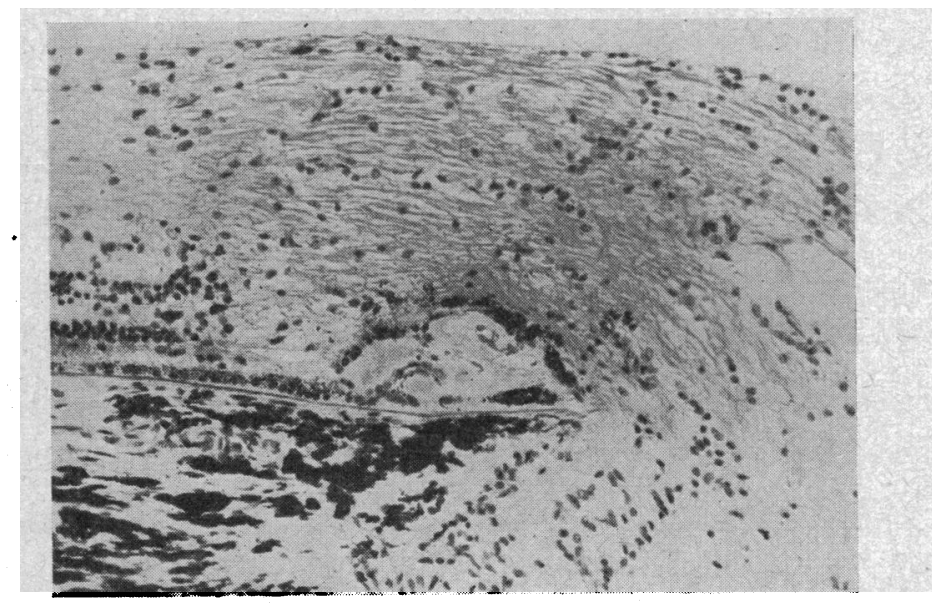

Fig. 1.

Vascularized colloid excrescence at margin of disc. The basophilic Bruch's membrane is also well shown. $\mathrm{H}$. and E. Photo: $\times 180$.

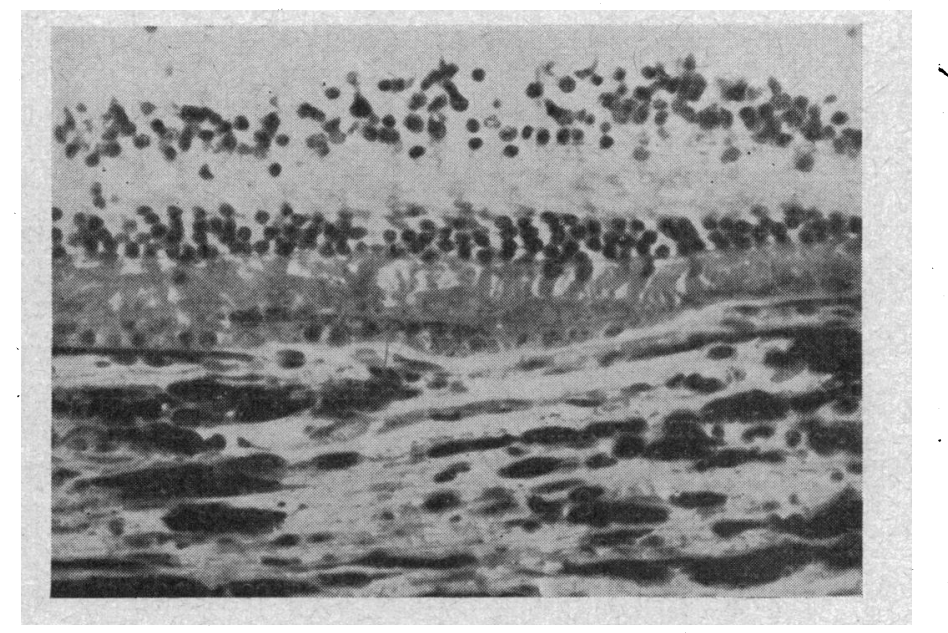

Fig. 2.

Wide gap in basophilic Bruch's membrane-oblique section of angioid streak. The pigment epithelium shows some post mortem disturbance and the chorio-capillaris is not well shown, but both are intact. A few new cells have been formed at the margins of the gap. Elastic tissue stain. Photo. $\times \mathbf{3 6 0}$. 
whole uvea and the sclera are more heavily pigmented than in the eye of a white person, but less so than in the eye of a pure-blooded negro.

Bruch's membrane in the posterior fundus stains strongly in haematoxylin, that is to say, is basophilic. The basophilia is continuous about half way to the equator on each side, then becomes patchy as in Fig. 2 of Verhoeff's and Sisson's paper, ${ }^{2}$ and finally ceases near the equator. However, even where it is continuous the basophilia is not uniform, but makes Bruch's membrane appear to vary considerably in thickness at frequent intervals. This indicates that originally the basophilia was patchy here as it is now towards the periphery. On the nasal side of the eye a wide gap is seen in the basophilic membrane in every section that includes the upper half of the optic disc. Reconstructed from the sections, the gap begins at the upper margin of the optic disc, courses nasally downward and reaches the horizontal mid-plane at a distance of $4 \mathrm{~mm}$. from the disc margin. Here the gap is $0.24 \mathrm{~mm}$. wide in the section. Below this level the gap is no longer seen, probably because it has become horizontal. In one place in the middle of the gap a short stretch of basophilic membrane remains, as if the membrane had ruptured in two places close together. At its narrowest place the gap is $0.06 \mathrm{~mm}$. wide in a section. In all sections the pigment epithelium and the choriocapillaris are intact at the site of the gap, and appear to be normal except that the epithelium shows slight post-mortem changes. In some sections these two structures are bulged slightly forward, in others slightly backward (Fig. 2) through the gap. This displacement is no doubt artificial. In a few sections there are within the gap several new formed spindle-cells just beneath the pigment epithelium (Fig. 2). Some of these are pigmented. The edges of the gap are not square, but always more - or less pointed, suggesting that the gap resulted from a rupture at a relatively weak place in the basophilic membrane.

In addition to the wide gap just described, two other gaps are found in Bruch's membrane. Each of these is very minute, only $0.009 \mathrm{~mm}$. wide, and is seen in only 4 consecutive sections. One is on the temporal side $0.45 \mathrm{~mm}$. from the disc, the other on the nasal side $1.0 \mathrm{~mm}$. from the disc (Fig. 3).

In the anterior part of the eye a pathological finding of possible importance is a localized exuberance of elastic tissue at the corneal limbus on each side in the horizontal meridian (Fig. 4). This elastic tissue is even more abundant than that generally found in a beginning pterygium, shows less degeneration than is usually found in pingueculae, and is not to any extent basophilic. 


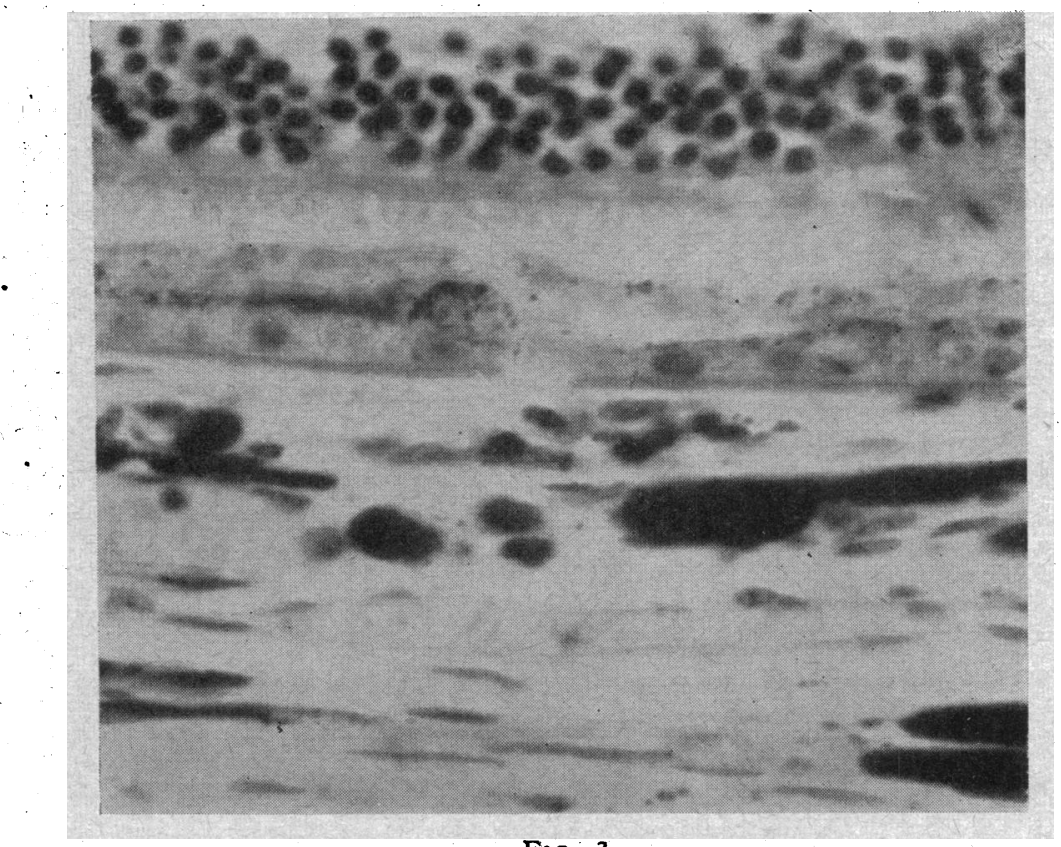

Fig. 3.

Minute gap in basophilic Bruch's membrane. H. and E. Photo. $\ .675$.

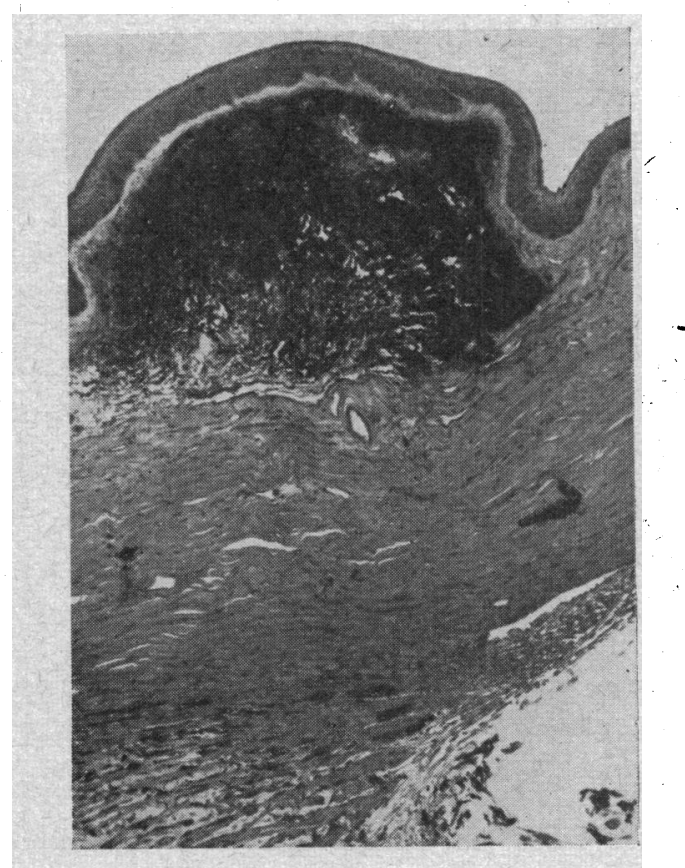

FIG. 4.

Exuberant elastic tissue (pinguecula) at corneal limbus, nasal side. Elastic tissue stain. Photo. $\times \mathbf{7 0}$. 


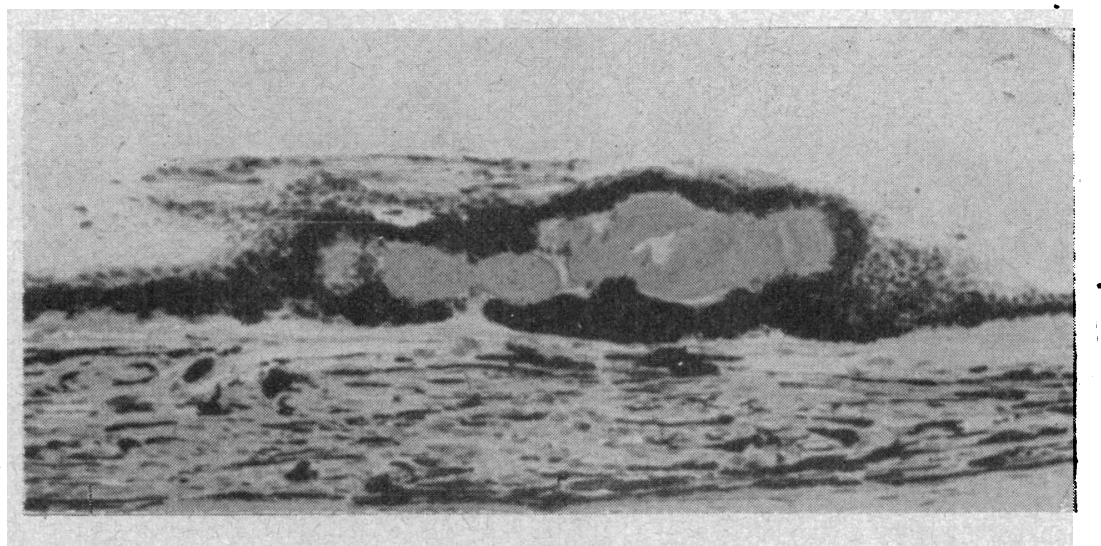

FIG. 5.

Concretion in epithelium of pars plana of ciliary body. A similar concretion was found in another section. H. and E. Photo. $\times 152$.

The cornea, iris, and lens are normal: The ciliary processes show hyaline change, consistent with the age of 50 years. Two sections some distance apart, each shows a fairly large concretion within the epithelium of the pars plana (Fig. 5). The sclera, including its elastic tissue, is normal and free from calcific deposits. The intra-ocular vessels, including those of the choroid and retina, show no definite sclerosis. Some of the choroidal arteries, owing to their post mortem collapse, appear to have unusually thick walls. The posterior ciliary vessels seen in the vicinity of the optic nerve are also free from sclerosis.

\section{Comment}

The only abnormal histological findings in the fundus of this eye, aside from the two drusen at the margin of the optic disc, were the basophilia of Bruch's membrane and the gaps in this membrane. Other than the wide gaps, seen on the nasal side in the sections, there was nothing to accounl for the angioid streak that had been seen ophthalmoscopically. It is especially noteworthy that the pigment epithelium and chorio-capillaris were intact at the site of the gap. Allowing for the fact that the streak was sectioned obliquely, its real width was probably about $0 \cdot 10$ $\mathrm{mm}$. to $0.05 \mathrm{~mm}$. The two other gaps found-were too minute to have been seen ophthalmoscopically, but probably in the course of time would have widened and lengthened. In other words, they probably indicated how angioid streaks begin.

To explain, on the basis of these findings, the ophthalmoscopic appearance of an angioid streak, one must assume that Bruch's 
membrane, especially when basophilic, obscures the choriocapillaris and contributes to the brightness of the fundus by reflecting a considerable amount of light. Within a gap, this reflection is absent, and the light is largely absorbed by the chorio-capillaris. Thus the appearance of a streak is due to increased visibility of the choroid, especially the dark red chorio-capillaris, exposed beneath the pigment epithelium at the site of a gap. This explains why in certain cases Dr. Clay has been able to obliterate the visibility of a streak by pressure upon the eye, for such pressure might express the blood from the chorio-capillaris. It is interesting that in the present case one of the sections in the middle of a gap showed an island such as occasionally is seen ophthalmoscopically in cases of angioid streaks. No doubt it resulted from Bruch's membrane rupturing at two places close together.

Böck $^{3}$, in 1938, reported the microscopic findings in two eyes, removed 48 hours post mortem, which ophthalmoscopically had shown numerous typical angioid streaks. Hagedoorn ${ }^{4}$, in 1939 , reported similar miscroscopic findings in two eyes also removed post mortem. In Hagedoorn's case the ophthalmoscopic examination was unsatisfactory, so that his microscopic findings would have been of doubtful significance were it not for the fact that they agreed with Böck's. Recently, Klien ${ }^{5}$ has reported the microscopic findings in two eyes in which angioid streaks had been found ophthalmoscopically. Her findings were essentially the same as those described by Hagedoorn. These observers noted that Bruch's membrane stained deeply in alum haematoxylin and found breaks in the membrane. Apparently none of them was aware of the findings of Verhoeff and Sisson ${ }^{2}$, noted below, although Hagedoorn used the term "basophilia" and found a senile eye in which this condition of Bruch's membrane existed. Böck thought that the gaps in the membrane would be ophthalmoscopically invisible if the pigment epithelium remained intact over them. Hagedoorn's explanation of the ophthalmoscopic appearance of the streaks was similar to that given above by me.

In 1928 I reported finding microscopically ${ }^{6}$, on the inner surface of the choroid of an enucleated eye, numerous small ridges. As seen microscopically these appeared as dark streaks similar in size and distribution to angioid streaks. In addition, I found changes in the macula that seemed to accord with those often seen ophthalmoscopically in cases of this condition. I felt sure, therefore, that the ridges were angioid streaks. Since such ridges were absent in Böck's, Hagedoorn's, Klien's, and the present case, I am compelled to abandon this view and to believe that my case was a highly unusual one in which the ophthalmoscopic picture would have closely simulated that of angioid streaks. It 
can now be regarded as certain that angioid streaks are gaps in an altered Bruch's membrane. Kofler ${ }^{7}$ astutely surmised this fact 27 years ago without histological evidence, but it was never generally accepted.

Many years ago I observed that in certain eyes Bruch's membrane stained deeply in alum haematoxylin, and in 1926 with Dr. Sisson made a special investigation of this phenomenon ${ }^{2}$. We found it in 60 out of 600 eyes selected at random, termed the condition of the membrane basophilia, and assumed it was due to calcification. Since we found it in no eye under 48 years of age, we concluded that it was a senile condition. We also concluded that it was not necessarily related to any other pathological condition in the eye.*

Bruch's membrane is generally described as consisting of two layers, an inner cuticular and an outer elastic layer. But behind the elastic layer, and also no doubt filling its interstices, is a thin layer of connective tissue which, as I have pointed out ${ }^{2}$, could be regarded as a third layer. Perhaps a more accurate way to describe Bruch's membrane is to say that it consists of an inner epiblastic cuticular layer produced by the pigment epithelium, and an outer mesoblastic layer composed of collagen abundantly pervaded in its inner portion by fine elastic fibrils. These fibrils are coarser near the optic disc and here extend entirely through the mesoblastic layer and continue into the underlying choroidal stroma. In thick cross sections, and often even in thin sections after formalin fixation, it is difficult or impossible to distinguish the two layers, the membrane appearing as a rather thick single layer. When the membrane is basophilic, this condition, if slight, often can be seen to involve only the elastic tissue, but if marked, it seems to involve the whole thickness of the membrane. Nevertheless even when the basophilia is marked, plane sections may show individual elastic fibrils. In its early stages the basophilia is not continuous, but is seen in cross sections in the form of long spindle-shaped spots resembling elongated nuclei. These appearances are depicted in the paper ${ }^{2}$ referred to.

In many eyes I have found Bruch's membrane throughout its thickness abnormally eosinophilic and hyaline, and not at all

\footnotetext{
* Mrs. Helenor Wilder has recently sent me from the Army Institute of Pathology in Washington sections of five eyes with such basophilia, from patients aged respectively $39,32,26,25$ and 20 years. In four of the eyes the basophilia was very slight and there were no breaks in the membrane. However, angioid streaks might have developed later. The other eye (aged 25 years) shrunken and degenerated as the result of an old injury, showed marked basophilia of Bruch's membrane and breaks indicative of angioid streaks. The possibility of angioid streaks existing in the fellow eye of this patient was not excluded. It is unlikely that the degeneration of this eye was an essential factor in the calcification of Bruch's membrane, since in many other similarly degenerated eyes $I$ have not found such calcification.
} 
basophilic. In some eyes it is both basophilic and eosinophilic, but I have not yet determined whether it is always eosinophilic when it is basophilic. However, I suspect this to be true and that in sections stained in both haematoxylin and eosin the basophilia hides the eosinophilia. I also suspect that basophilia is always preceded by eosinophilia of Bruch's membrane. In the present case, in sections that are stained only lightly in haematoxylin it is easily recognizable that Bruch's membrane is both eosinophilic and basophilic. Unna called degenerated elastic tissue which stained in haematoxylin, elacin. It has been found this gives the differential staining reactions of calcium, and often in addition those of iron. It is evident, therefore, that basophilia of Bruch's membrane means calcification of this membrane as I originally assumed. The basophilia of elacin elsewhere, however, is seldom if ever as intense as it often is in Bruch's membrane. Böck found that in his case the membrane stained differentially for calcium but not for iron. Hagedoorn found that it stained differentially for iron but not for calcium and attributed failure of the calcium test to acidity of the formalin used in the fixation. Klien found that the membrane gave the Kossa reaction for calcium and the Turnbull blue reaction for iron.

That basophilia of Bruch's membrane sometimes occurs in senile eyes is not surprising, for degeneration and calcification are characteristic of old age. But according to $\mathrm{Holloway}^{8}$ almost half of the cases of angioid streaks have been observed before the age of 40 , and 4 cases before the age of 20 . Since vision is not appreciably impaired by the condition until the onset of macular changes, no doubt many of the cases were not discovered until long after streaks had.formed. Hence in cases of angioid streaks, basophilia of Bruch's membrane is not dependent upon age. Nor is it necessarily dependent upon defective calcium metabolism, for degenerated tissues readily undergo calcification even in young normal individuals. Hagedoorn assumed that the basophilia was due to "precocious senility of the elastic fibres" of the body.

Two closely related questions now arise. First, what causes gaps in basophilic membrane? Second, why are angioid streaks not associated with the basophilia that often occurs in senile eyes? The second question was not discussed by Böck, Hagedoorn, or Klien, evidently because they were unaware of the fact that basophilia of Bruch's membrane is a common senile condition. Theoretically a gap could be caused by complete dissolution of Bruch's membrane at a localized site. But microscopically there is no evidence for this. It seems certain that the membrane has ruptured and that the broken edges have separated, leaving the 
other tissues apparently unaltered. Assuming, as also seems certain, that the basophilia is due to calcification, it is obvious that a basophilic membrane could be fractured by voluntary movements of the eye, winking, and " rubbing the eye" by the hands. Widening of the gaps probably results not from elasticity of the membrane, because calcification would do away with its elasticity, but from the elasticity of the choroid as a whole. Presumably this elastic tension not only widens and extends the gaps but contributed to their original cause. In senile eyes, it is reasonable to suppose that the choroid has largely lost its elasticity long before the membrane is calcified. Hence angioid streaks do not usually result when fractures of Bruch's membrane occur in senile eyes-the broken edges do not tend to separate and may even tend to override each other (Fig. 1, Verhoeff and Sisson ${ }^{2}$ ). That angioid streaks occur chiefly in the vicinity of the optic disc is no doubt explained by the fact that basophilia is always most marked here. This may be because, as I have already pointed out, Bruch's membrane is normally somewhat different in this vicinity than elsewhere. Why, in cases of angioid streaks, and in many senile eyes, this membrane alone of all structures of the choroid should undergo calcification is not obvious. Possibly this fact is partly dependent upon the close relation of the membrane to the chorio-capillaris.

A feature characteristically associated with angioid streaks is a mottled appearance of the fundus. Probably this is due to unevenness and discontinuities such as seen microscopically in the basophilia of Bruch's membrane in the present case. The marked proliferative changes within and along the gaps in three of the cases explain the white borders and ophthalmoscopic appearances of some of the older streaks. Such proliferative changes may also explain the disappearance of streaks. In none of these cases was there any reformation of Bruch's membrane.

Haemorrhages described as choroidal or sub-retinal are frequently seen in cases of angioid streaks. Since the haemorrhages often occur early in life, it seems to me probable that they are usually not due to local vascular disease, but to fracture of the calcified Bruch's membrane causing rupture of the choriocapillaris. In some cases the rupture and haemorrhage might not result immediately'from the fracture, but later from widening of a streak. If the pigment epithelium was not ruptured, the blood could lift it up in the form of a mound and later become organized into solid tissue. When occurring beneath the macula this process would result in the condition known as disciform degeneration of the macula. As first demonstrated by Verhoeff and Grossman ${ }^{9}$ and soon afterwards but less conclusively by Braun ${ }^{10}$, 
senile disciform degeneration of the macula is typically produced by haemorrhage beneath the pigment epithelium.

In addition to the streaks, mottling of the fundus, and subretinal haemorrhages, other fundus changes often occur in cases of angioid streaks. It is believed that macular changes will ultimately occur in every-case if the patient lives long enough. Only exceptionally do these macular changes take the form of the typical disciform degeneration just mentioned. In the present case the fundi, including the maculae, were normal except for the streaks. This was true also of Böck's case, with the exception that there was some slight formation of new tissue on Bruch's membrane elsewhere than in the macula. Only Hagedoorn's and Klien's cases afford histological information as to the late fundus changes. 'The findings in their cases were remarkably alike. In each, both maculae were involved, and in similar fashion-there was a thick layer of vascularized tissue on the inner surface of Bruch's membrane. This tissue was very old and in each case showed foci of ossification in one eye. In both cases similar tissue was also present elsewhere in the fundi. Klien described in addition, the widespread formation of a thick cuticular layer by the pigment epithelium. On the basis of her histological findings she satisfactorily accounts for the fundus pictures in five clinical cases of angioid streaks.

Aside from the streaks themselves, and possibly the deeply seated haemorrhages, none of the fundus changes seen in cases of angioid streaks is peculiar to this condition. Thus the vascularized tissue, found by Hagedoorn and Klien between Bruch's membrane and the pigment epithelium, may occur in eyes without angioid streaks, and without basophilia of Bruch's membrane. Most commonly it occurs in senile eyes, but is not necessarily associated with local vascular changes. Brown ${ }^{11}$ has called special attention to it. Vessels enter this tissue through holes in the membrane. These holes are not due to fractures of the membrane, for usually it is not basophilic. I assume that they result from penetration of the membrane by capillary sprouts which develop into arteries and veins. Sometimes this tissue is formed beneath the macula and produces a condition that ophthalmoscopically would be difficult to distinguish from the typical senile disciform degeneration due to haemorrhage. In fact, in Hagedoorn's and Klien's cases I am unable to decide this question even by the microscopical evidence.

Since in cases of angioid streaks, cardio-vascular disease often develops, it is probable that in some of the cases disciform degeneration of the macula is dependent upon vascular disease just as it usually is in ordinary senile cases. Haemorrhages 
within the retina, and circinate retinitis, occurring in association with angioid streaks, are no doubt due to vascular disease. Klien attributes almost if not all the changes in the fundi in cases of angioid streaks to calcification of Bruch's membrane. Thus she says " The clinical picture of angioid streaks represents not only the visible ruptures in Bruch's membrane but also the multiform end results of irritation of pigment epithelium and chorio-capillaris by the sharp, calcified edges and fragments of the broken membrane." With this view I cannot fully agree. For as already indicated I have seen in eyes showing no basophilia of Bruch's membrane the changes she attributes to irritation by calcified membrane. Among these changes she does not mention subretinal haemorrhage, which, as I have suggested above, may result from fracture of the calcified membrane.

In the present case at the margin of the disc there were two large " colloid excrescences" ("Drusen "), one of them vascularized. Böck and Hagedoorn also noted "Drusen" on the discs in their cases. Such formations are typically senile, that is to say degenerative in origin, but it is impossible to maintain that degeneration of elastic tissue has anything to do with them. Klien found "several hyaline bodies in the temporal half of the left nervehead." It would seem, therefore, that in cases of angioid streaks there is not only degeneration of Bruch's membrane, but sooner or later also degeneration of the pigment epithelium and various proliferative changes associated therewith. A degenerative process noted in the present case but not in the other three cases, was the formation of concretions in the pars ciliaris retinae (Fig. 5).

On each side of the eye examined in the present case there was a large pinguecula. Hagedoorn noted a large pinguecula on one eye in his case. Böck and Klien noted no pingueculae in their , cases, but may not have looked for them. As a matter of fact, a section kindly sent me by Dr. Klien shows a pinguecula on the nasal side of the left eye-she did not section the anterior part of the right eye. Histologically, a pinguecula except in size is essentially identical with a lesion of pseudo-xanthoma elasticum. But since both conditions are similar to senile elastices of the skin the only relationship between them may be that both are degenerative conditions.

It is noteworthy that in all four of the cases in which the eyes have been examined microscopically, death was due to cardiovascular disease. In Böck's case the patient was a male, aged 44, in Hagedoorn's a female, aged 48, in Klien's a male, aged 53, and in the présent case a female, aged 50. Pseudo-xanthoma 
elasticum was absent only in Klien's case. In the present case, although there were evidences of marked general arteriosclerosis of the larger vessels, the vessels of the retina and choroid, and the posterior ciliary vessels seen in the sections, showed no definite sclerosis. Böck also found, with the exception of one choroidal artery, the retinal and choroidal vessels free from sclerosis, but found marked sclerosis of the posterior ciliary arteries. He thought that the atheromatous changes in the aorta in his case were primarily due to degeneration of elastic tissue. Hagedoorn in his case found slight thickening of the walls of the choroidal arteries but no intimal proliferation. No autopsy was done in his case. Klien's findings were substantially the same as Hagedoorn's. It would seem that in none of the four cases were vascular changes in the choroid or retina sufficiently marked to account for any other lesions in the eyes.

There are three conditions that have been associated with angioid stręaks too often to be merely coincidental, namely, cardio-vascular disease, pseudo-xanthoma elasticum, and Paget's disease. Holloway ${ }^{8}$ found that cardio-vascular disease was noted in 23.3 per cent. of the cases he collected from the literature. Actually it may have been overlooked in many of the other cases. This fact suggests that vascular disease will finally develop, and at a relatively early age, in every case of angioid streaks. Clay ${ }^{12}$ found pseudo-xanthoma elasticum in 30 per cent. of his cases of angioid streaks. Apparently I was the first to note angioid streaks in a case of Paget's disease ${ }^{6}$. Twelve such cases have been reported to date but these may give an inadequate idea of the frequency of the association, for Terry ${ }^{13}$ found three cases of angioid streaks in 22 cases of Paget's disease. Paget's disease is typically associated with general arteriosclerosis, but no case has been reported in which it was associated with both angioid streaks and pseudo-xanthoma elasticum.

It requires great stretching of the imagination to assume a common systemic cause for angioid streaks, cardio-vascular disease, pseudo-xanthoma elasticum, and Paget's disease. It is true that some of the elastic tissue in pseudo-xanthoma becomes basophilic, but there is first marked hyperplasia of the elastic tissue and this does not occur in the case of Bruch's membrane. As regards Paget's disease, Klien suggests that in this disease the angioid streaks may have a different origin, in that calcium is deposited in a normal Bruch's membrane. I can explain the associations in question only by assuming that the four conditions are independently abiotrophic and when they occur together do so as the result of hereditary linkages. 


\section{Conclusions.}

Angioid streaks result from ruptures of a basophilic Bruch's membrane. The gaps are widened by the elastic tension of the choroid. In the ophthalmoscopic picture the basophilic condition (calcification) brightens the contiguous fundus and makes conspicuous the dark red chorio-capillaris exposed beneath the pigment epithelium by the ruptures. The streaks are later often altered in appearance by proliferative changes along or within them. The similar ruptures that occasionally occur in senile eyes do not widen into visible streaks, probably because the senile choroid loses its elasticity before Bruch's membrane becomes calcified.

Other lesions that occur in the macula and elsewhere in the fundus in cases of angioid streaks are usually due to proliferative changes involving the pigment epithelium, such as occur under senile degenerative conditions. The subretinal haemorrhages in the macula and elsewhere may be due to fractures of the calcified membrane causing ruptures of the chorio-capillaris. When such a haemorrhage lifts up the pigment epithelium in the macula, " disciform degeneration" results. Haemorrhages arising within the retina, are probably explained as is also circinate retinitis, by the vascular changes that commonly occur in cases of angioid streaks.

The association of angioid streaks in many cases with pseudoxanthoma elasticum, in some cases with Paget's disease instead, and in still other cases with neither of these conditions is difficult to explain, as is also their association with cardio-vascular disease. A possible explanation is that these associations are dependent upon hereditary linkages.

\section{REFERENCES}

1. Verhoeff, F. H.-An improved differential elastic tissue stain. Jl. A.M.A., Vol. LVI, p. 1326, 1911.

2. VerhoefF, F. H., and Sisson, R. J.-Basophilic staining of Bruch's membrane. Arch. Ophthal., Vol. LV, p. 125, 1926.

3. Böck, J,-Zur Klink und Anatomie der gefassahmlichen Streifen in Augenhintergrund. Zeitsch.f. Augenheilk., Vol. XCV, p. 1, 1938.

4. Hagedoorn, A.-Angioid Streaks. Arch. Ophthal., Vol. XXI, p. 746, 1939.

5. Klien, Bertha A.—Angioid Streaks. Amer. Jl. Ophthal., Vol. XXX, p. 955, 1947.

6. VERHOEFF, F. H.-The nature and pathogenesis of angioid streaks in the ocular fundus. Trans. Ophthal. Sec. A.M.A., p. 243, 1928.

7. Kofler, A.-Beitrage Zur Kenntnis der Angioid Streaks. Arch. f. Augenheilk., Vol. LXXXII, p. 134, 1917.

8. Holloway, T. B.-Angioid Streaks; A report concerning two cases. Trans. Amer. Ophthal. Soc., p. 173, 1927.

9. Verhoeff, F. H., and Grossman, H. P.-Pathogenesis of disciform degeneration of the macula. Arch. Ophthal, Vol. XVIII, p. 561, 1937.

10. BRAUN, ReINHARD. - Pathologisch-Anatomischer Beitrag zur Frage der Scheibenformigen Entärtung der Fundusmitte. Arch. f. Augenheilk., Vol, CX, p. 535, 1937.

11. Brown, E. V. L.-Retroretinal tissue from the choroid in Kuhnt-Junius degeneration of the macula. Arch. of Ophthal., Vol. XXIII, p. 1157, 1940.

12. Clay, Grady E.-Discussion of Bedell's paper, " Angiod streaks in the deep layers of the retina," Trans. Amer. Ophthal. Soc., p. 302, 1944.

13. Terry, T. L.-Angioid streaks and osteitis deformans. Trans. Amer. Ophthal. Soc., p. 555, 1934. 